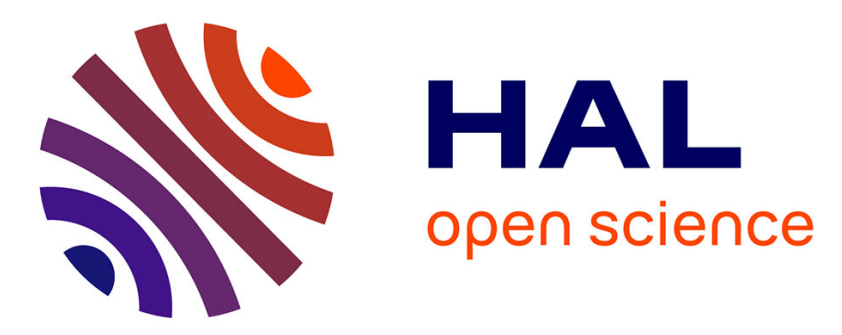

\title{
Human ornithine decarboxylase-paralogue (ODCp) is an antizyme inhibitor but not an arginine decarboxylase
} Kristiina Kanerva, Laura T. Mäkitie, Anna Pelander, Marja Heiskala, Leif C. Andersson

\section{- To cite this version:}

Kristiina Kanerva, Laura T. Mäkitie, Anna Pelander, Marja Heiskala, Leif C. Andersson. Human ornithine decarboxylase-paralogue (ODCp) is an antizyme inhibitor but not an arginine decarboxylase. Biochemical Journal, 2007, 409 (1), pp.187-192. 10.1042/BJ20071004 . hal-00478855

\section{HAL Id: hal-00478855 https://hal.science/hal-00478855}

Submitted on 30 Apr 2010

HAL is a multi-disciplinary open access archive for the deposit and dissemination of scientific research documents, whether they are published or not. The documents may come from teaching and research institutions in France or abroad, or from public or private research centers.
L'archive ouverte pluridisciplinaire HAL, est destinée au dépôt et à la diffusion de documents scientifiques de niveau recherche, publiés ou non, émanant des établissements d'enseignement et de recherche français ou étrangers, des laboratoires publics ou privés. 


\section{Human ornithine decarboxylase-paralogue (ODCp) is an antizyme inhibitor but not an arginine decarboxylase}

Kristiina Kanerva*, Laura T. Mäkitie*, Anna Pelander†, Marja Heiskalał, Leif C. Andersson§

*Haartman Institute, University of Helsinki, Department of Pathology, Helsinki, Finland

$\dagger$ University of Helsinki, Department of Forensic Medicine, Helsinki, Finland ¥Schering-Plough Research Institute, Kenilworth, NJ 07033, USA

$\S$ Haartman Institute, University of Helsinki and HUSLAB, Department of Pathology, Helsinki, Finland

Correspondence to: Leif C. Andersson, Dept. of Pathology, Haartman Institute, P.O.

Box 21 (Haartmaninkatu 3), FIN-00014 University of Helsinki, Finland

Phone: +358-9-19126510; Fax: +358-9-19126675;

E-mail: Leif.Andersson@helsinki.fi

Running title: ODCp is an antizyme inhibitor

Abbreviations used: ODCp, ornithine decarboxylase-paralogue; ODC, ornithine decarboxylase; ADC, arginine decarboxylase; AZI, antizyme inhibitor; AZ, antizyme; GST, glutathione $S$-transferase; F-II, rabbit reticulocyte lysate fraction II. 


\begin{abstract}
Ornithine decarboxylase (ODC), the rate-limiting enzyme in polyamine biosynthesis, is regulated by specific inhibitors, antizymes (AZ), which in turn are inhibited by antizyme inhibitor (AZI). We originally identified and cloned the cDNA for a novel human ODC-like protein called ornithine decarboxylase-paralogue (ODCp). Since ODCp was devoid of ODC catalytic activity, we proposed that ODCp is a novel form of AZI. ODCp has subsequently been suggested to function either as mammalian arginine decarboxylase (ADC) or as AZI in murine. Here we report that human ODCp is a novel AZI (AZI2). By using yeast two-hybrid screening and in vitro binding assay we show that ODCp binds AZ1-3. Measurements of ODC activity and ODC degradation assay reveal that ODCp inhibits AZ1 function as efficiently as AZI both in vitro and in vivo. We further demonstrate that the degradation of ODCp is ubiquitin-dependent and AZ1-independent similar to the degradation of AZI. We also show that human ODCp has no intrinsic ADC activity.
\end{abstract}

Key words: Ornithine decarboxylase (ODC), ODC-paralogue (ODCp), Antizyme inhibitor (AZI), Arginine decarboxylase (ADC), Antizyme (AZ), Polyamine 


\section{INTRODUCTION}

We originally identified a novel human ornithine decarboxylase-like protein called ornithine decarboxylase-paralogue (ODCp) [1]. The odcp gene encodes a $50 \mathrm{kDa}$ protein comprising several motifs and domains conserved in ornithine decarboxylase (ODC), including pyridoxal-5-phosphate (PLP) binding site, membrane translocation motif $\mathrm{p} 47^{\text {phox }}[2]$ and antizyme (AZ) binding site [1,3]. The key residues important for the decarboxylase activity of ODC are, however, mutated in ODCp, as is the PEST domain essential for the rapid turnover of ODC. Therefore we suggested that ODCp is a novel tissue-specific antizyme inhibitor (AZI) [1]. The tissue distribution of ODCp is restricted, with high expression in brain and testis.

Two different roles have subsequently been proposed for ODCp. Zhu et al. reported that ODCp is a mammalian arginine decarboxylase (ADC), which catalyses the decarboxylation of L-arginine to agmatine [4]. Micro-organisms and plants contain $\mathrm{ADC}$, and they produce putrescine from both agmatine and ornithine [5, 6]. In mammals that receive agmatine from their diet, the existence of ADC has not been demonstrated previously. Later studies have, however, been unable to verify the ADC activity of ODCp [7, 8]. López-Contreras et al. recently reported that the murine paralogue of ODCp acts as an AZI [8]. They showed that interaction between AZ1-3 and mouse ODCp leads to increased ODC activity and decreased rate of ODC degradation.

The family of AZs in mammals includes at least three members. AZ1 and AZ2 are expressed ubiquitously in all cell types, but the expression levels of AZ2 are lower [9]. AZ3 is a testis-specific paralogue, the expression of which is restricted to certain stages of spermatogenesis; in late spermatocytes and early spermatids [10]. All AZs have been shown to inhibit the decarboxylase activity of ODC [10-12]. AZ1 is also able to induce ODC degradation via $26 \mathrm{~S}$ proteasomes but it is still unclear whether the other AZs display this function [8, 11]. All AZs are inactivated by AZI [12], a protein which displays sequence homology to ODC, but lacks decarboxylating activity [13]. AZI binds AZs with higher affinity than does ODC, and releases active ODC from the catalytically inactive ODC-AZ complex [14]. 
In this study we addressed the question of whether human ODCp functions as AZI or ADC. Here we report that ODCp acts as a regulator of ODC activity and inhibits its proteasomal degradation. ODCp is degraded by ubiquitination like AZI. We also demonstrate that ODCp does not act as mammalian ADC. Our results reveal that human ODCp is a novel AZI.

\section{EXPERIMENTAL}

\section{Materials}

Oligonucleotides were purchased from Sigma-Proligo. Creatine phosphate was obtained from Fluka, and isopropyl- $\beta$-D-thiogalactopyranoside (IPTG) from Fermentas. All the other chemicals were obtained from Sigma.

\section{Plasmid construction}

The cDNAs encoding ODCp, AZ1 and AZ3 were amplified from human QUICK Clone cDNA libraries (Clontech) using whole brain library as a template for AZ1 and ODCp, and a testis library for AZ3. IMAGE cDNA clones from the RZPD German Resource Center for Genome Research were used as templates for the amplification of human AZ2 (clone ID IRAKp961E02134Q2) and AZI (clone ID IRAUp969Bo377D6). To clone AZs, the ribosomal frame-shifting site was deleted as described previously [12]. The resulting AZ1-3 fragments, AZI and ODCp were subcloned into the pCDNA3.1 vector (Invitrogen) under the control of the T7 promoter. The human ODC cDNA (a kind gift from Dr. Erkki Hölttä) was cloned into the pCIneo vector (Promega). For transient transfection experiments ODCp and ODC cDNAs were cloned into the p3xFLAG-CMV-10 vector (Sigma), and AZI into the pAMC expression vector (a generous gift from Dr. Tatiana Petrova) to produce Nterminally c-Myc tagged AZI.

\section{Cell culture and transient transfections}

COS-7 cells were cultured in Dulbecco's modified Eagle's medium (DMEM) supplemented with $10 \%$ fetal bovine serum (FBS), $1 \mathrm{mM}$ L-glutamine, $50 \mathrm{mg} / \mathrm{ml}$ penicillin and $50 \mathrm{mg} / \mathrm{ml}$ streptomycin. Transient transfections of COS-7 cells, used in 
ODC activity assay, were performed with FuGENE HD (Roche). For ADC activity measurements COS-7 cells were transfected with Lipofectamine ${ }^{\mathrm{TM}} 2000$ (Invitrogen) according to the manufacturer's instructions.

\section{Yeast two-hybrid screening}

The yeast two-hybrid screening was performed using Matchmaker ${ }^{\mathrm{TM}}$ Two-hybrid System 3 (Clontech). Briefly, the full-length human ODCp cDNA was cloned inframe with GAL4 DNA-binding-domain of pGBKT7 vector and used as a bait construct for the analysis. The bait was transformed into yeast strain AH109, which was mated to strain Y187 carrying the prey library (pretransformed human bone marrow MATCHMAKER cDNA library, catalogue number HY4053AH, Clontech) in pACT2 vector. No autonomous activation of the reporter genes was observed with the bait construct. The positive clones on selective media were further confirmed by monitoring expression of the reporter genes, and the inserts of the positive clones were amplified by PCR and sequenced.

\section{In vitro translation}

L- $\left[{ }^{35} \mathrm{~S}\right]$ methionine (Amersham Biosciences) labeled AZ1-3, AZI, ODCp, and ODC were synthesized in vitro with the T7 TNT system (Promega) from the corresponding pCDNA3.1 and pCIneo constructs. The protein amounts of different in vitro translation samples were determined and equalized prior assays by SDS-PAGE and fluorography.

\section{GST fusion proteins and pull-down assay}

The full-length human ODCp was cloned into the pGEX-4T-3 vector (Amersham Biosciences). GST and GST-ODCp fusion proteins were expressed in E. coli BL21. The fusion proteins were purified from crude bacterial lysates with glutathione sepharose 4B beads (Amersham Biosciences) and analyzed by Western blotting, using the anti-GST antibody (Serotec) to equalize the protein amounts. Glutathione sepharose-bound GST or GST-ODCp were incubated with in vitro translation products in binding buffer $(10 \mathrm{mM}$ Tris- $\mathrm{HCl}, \mathrm{pH} 7.5,150 \mathrm{mM} \mathrm{NaCl}, 1 \mathrm{mM}$ EDTA, $1 \%$ Triton X-100, protease inhibitors) at RT. After $1 \mathrm{~h}$, the beads were washed at least 
five times with binding buffer. The bound proteins were eluated in Laemmli sample buffer and resolved on a 13\% SDS-PAGE and visualized by fluorography.

\section{In vitro protein degradation assay}

In vitro translated $\left[{ }^{35} \mathrm{~S}\right]$ methionine-labeled proteins were combined in proportions 2:1:14 (ODC:AZ1:ODCp/AZI) and incubated in a reticulocyte lysate-based degradation mixture with ATP-regenerating buffer $(50 \mathrm{mM}$ Tris-HCl, $\mathrm{pH} 7.5,5 \mathrm{mM}$ $\mathrm{MgCl}_{2}, 2 \mathrm{mM}$ dithiothreitol, $0.5 \mathrm{mM}$ ATP, $10 \mathrm{mM}$ creatine phosphate, $1.6 \mu \mathrm{g} / \mathrm{ml}$ phosphocreatine kinase) at $37^{\circ} \mathrm{C}$ for the indicated times. Equal amounts of aliquots of the reaction were stopped by addition of Laemmli sample buffer. The amount of proteins remaining undegraded was determined by SDS-PAGE and fluorography. The intensities of the bands were analyzed also by scanning the dried, fluorographed gel with Phosphoimager (BAS-1500, Fuji). In the in vitro degradation assay with fraction II, $10 \mu \mathrm{l}$ of buffer was replaced by fraction II of rabbit reticulocyte (Calbiochem). Otherwise the assay was performed as mentioned above.

\section{Ubiquitin conjugation assay}

In vitro translated $\left[{ }^{35} \mathrm{~S}\right]$ methionine-labeled AZI, ODCp, and ODC were incubated at $37{ }^{\circ} \mathrm{C}$ for the indicated times in reaction buffer $(20 \mathrm{mM}$ Tris- $\mathrm{HCl}, \mathrm{pH} 7.5,5 \mathrm{mM}$ $\mathrm{MgCl}_{2}, 2 \mathrm{mM}$ dithiothreitol, $0.5 \mathrm{mM}$ ATP, $0.5 \mu \mathrm{g}$ ubiquitin aldehyde, $5 \mu \mathrm{g}$ ubiquitin). The degradation of equal-sized aliquots was determined by SDS-PAGE, fluorography and Phosphoimager scanning. When the accumulation of high molecular weight ubiquitin conjugates of the proteins were analyzed, proteasome inhibitors $(150 \mu \mathrm{M}$ MG132 and $150 \mu \mathrm{M}$ clasto-Lactacystin $\beta$-lactone) were added in the reaction buffer.

\section{Ornithine decarboxylase assay}

The ODC activity was determined by measuring the release of ${ }^{14} \mathrm{CO}_{2}$ from L-[1${ }^{14} \mathrm{C}$ ]ornithine as described previously [1]. For the assay, in vitro translated proteins were combined in proportions 2.5:1:7 (ODC:AZ1:ODCp/AZI), and the assay was repeated three times. To measure the ODC activity in vivo, COS-7 cells were transiently transfected with FLAG-ODCp, pAMC-AZI or the empty p3xFLAGCMV-10 vector. After $24 \mathrm{~h}$, the cells were collected as described earlier [1], and ODC 
activity was measured. Transfection experiment was performed in duplicate, and all the measurements were performed as triplicate.

\section{Arginine decarboxylase assay}

The ADC activity measurement was performed essentially as described previously [4]. $10 \mu \mathrm{l}$ of in vitro translated ODCp and ODC were diluted directly to the ADC assay reaction buffer whose $\mathrm{pH}$ was either 7.4 or 8.25 . In the former $\mathrm{pH}$, the assay was carried out at $37^{\circ} \mathrm{C}$ and in the latter at $30^{\circ} \mathrm{C}$. The empty pCDNA3.1 vector was used as a negative control and E. coli $\operatorname{ADC}(0,021 \mathrm{U} /$ reaction, Sigma) as a positive control. The assay was repeated three times in both conditions. COS-7 cells, transfected with FLAG-ODCp, FLAG-ODC, or p3xFLAG-CMV-10 vector, were lysed in the sample buffer (10 mM Tris- $\mathrm{HCl}, \mathrm{pH} 7.4$ or 8.25 , protease inhibitors) by sonicating, and centrifuged at $1000 \mathrm{~g}$ for $10 \mathrm{~min}$ at $4{ }^{\circ} \mathrm{C}$. The supernatant was recentrifuged at $12000 \mathrm{~g}$ for $20 \mathrm{~min}$ at $4{ }^{\circ} \mathrm{C}$ to obtain cytosolic and crude mitochondrial fractions. The mitochondrial pellet was resuspended in the sample buffer ( $\mathrm{pH}$ 8.25). The ADC assay of the cytosolic fraction was performed in both conditions mentioned above but the mitochondrial fraction was analyzed only in $\mathrm{pH}$ 8.25 at $30{ }^{\circ} \mathrm{C}$. All the measurement were performed as triplicate, and the expression of ODC and ODCp was verified by Western blotting with anti-FLAG M2 antibody.

\section{Measurement of agmatine by chromatography and mass-spectrometry}

ODCp was expressed in COS-7 cells for 24-36 h. Untransfected cells cultured for $2 \mathrm{~h}$ in the presence of $10 \mathrm{mM}$ agmatine were used as a positive control. Agmatine analysis was performed by electrospray ionization (ESI) liquid chromatography timeof-flight mass spectrometry (LC/TOFMS). The identification of agmatine was based on accurate mass determination. For mass spectrometry, the dialyzed samples were diluted in $0.1 \%$ formic acid-acetonitrile. The liquid chromatograph was an Agilent (Waldbronn) 1100 series instrument. The separation was performed with a Phenomenex (Torrance) Luna C-18(2) $100 \times 2 \mathrm{~mm}(3 \mu \mathrm{m})$ column with a $21 \mathrm{~min}$ gradient at $40{ }^{\circ} \mathrm{C}$ at a flow rate of $0.3 \mathrm{ml} / \mathrm{min}$. The eluent components were $5 \mathrm{mM}$ ammonium acetate in $0.1 \%$ formic acid and acetonitrile. The injection volume was 20 $\mu 1$. The mass analyzer was a Bruker Daltonics (Bremen) MicrOTOF with an orthogonal ESI source operated in the positive ion mode with a $m / z$ range of $50-800$. 
The capillary exit voltage was set at $70 \mathrm{~V}$, the hexapole RF at $50 \mathrm{~V}$, and the transfer time at $35 \mu \mathrm{s}$. The nebulizer gas flow was $1.6 \mathrm{bar}$, the drying gas flow was $8 \mathrm{l} / \mathrm{min}$, and the drying temperature was $200{ }^{\circ} \mathrm{C}$. The external and post-run calibrations were performed with sodium formate clusters in quadratic mode with six calibration points.

\section{RESULTS AND DISCUSSION}

\section{Human ODCp interacts with AZ1, AZ2, and AZ3}

We screened a yeast two-hybrid library of human bone marrow cDNA using the fulllength human ODCp as a bait. Twenty six clones were isolated and sequenced. A GenBank search revealed that 21 of these clones encoded human ornithine decarboxylase antizyme 1 (GenBank accession no. NM_004152). The interaction between ODCp and AZ1 was further confirmed by the GST pull-down assay. GST pull-down was also used to test whether ODCp interacts with AZ2-3. In vitro translated, ${ }^{35}$ S-labeled AZ1-3 were incubated with immobilized GST or GST-ODCp. AZ1, AZ2, and AZ3 bound to GST-ODCp fusion protein, but not to GST alone (Figure 1).

The binding of ODCp to AZ1-3 thus supported our original hypothesis that ODCp is a tissue-specific AZI [1]. Moreover, Kidron et al. recently reported a close relationship between ODCp and AZI sequences in the phylogenetic tree [3]. They also showed that human ODCp is not significantly homologous to ADCs of other species, and concluded that it is very unlikely that human ODCp could account for any ADC activity. Our results are also in agreement with the report of López-Contreras et al. who, using co-immunoprecipitation, showed that mouse ODCp interacts with AZ1-3 [8]. We used a different method to show the interaction between ODCp and AZ1-3, and actually two independent methods confirmed that ODCp binds to AZ1. Our results thus demonstrate that human ODCp binds AZ1-3, as does the mouse orthologue of ODCp.

\section{Human ODCp acts as an AZI}

To study the AZI activity of human ODCp, we combined in vitro translated ODC

with AZ1 and AZI or ODCp, and measured the ODC activity of the reactions. We 
also measured the activity of AZI and ODCp alone to ensure that they by themselves did not contribute to ODC activity. As shown in Figure 2 A, ODC activity increased in the reaction mixtures containing ODC with either ODCp or AZI together with AZ1, compared with the control reaction containing ODC with AZ1. This indicates that in vitro human ODCp functions as an AZI. To investigate this possibility also in vivo, we transfected COS-7 cells with cDNA encoding FLAG-ODCp, c-Myc-AZI or empty p3xFLAG-CMV-10 vector, after which the ODC activity was measured. In the cells transfected with ODCp, a rise in ODC activity similar to that observed in the AZI transfectants took place (Figure 2 B). Our findings suggest that ODCp activates ODC at least as efficiently as AZI. In our study, the increase in ODC activity was in fact slightly higher in the ODCp transfectants than in the AZI transfectants. This might be due to a difference in expression level of ODCp and AZI. Nevertheless, it would be interesting to further examine whether ODCp acts more efficiently in activation of ODC than AZI.

The ODCp-induced activation of ODC does not by itself prove that ODCp acts as an AZI. The interaction of ODCp with AZ1 could also have other functionality than regulation of ODC activity since it has been shown that AZ1 can interact and regulate several additional proteins besides ODC $[15,16]$. Since there is only a limited amount of AZ1 molecules in the reaction mixture, ODCp could sequester all AZ1, thereby competing with ODC for AZ1 binding without still having AZI activity as a main function itself. To ultimately function as AZI, ODCp should also inhibit the AZ1dependent degradation of ODC, but on the other hand, the interaction between ODCp and AZ1 should not induce degradation of ODCp itself. To examine whether ODCp competes with ODC for the binding of AZ1 and inhibits ODC degradation, we used an in vitro protein degradation assay with rabbit reticulocyte lysate as a source of proteasomes. As reported earlier by Li and Coffino [17], ODC alone is not degraded in reticulocyte lysate in time frame used in the experiment, whereas in the presence of AZ1 the degradation occurs rapidly (Figure 3 A). When AZI or ODCp were added to the reaction, the AZ1-induced degradation of ODC was prevented. We noted that the in vitro degradation assay was sensitive to the relative amounts of the proteins. Hence, López-Contreras et al. did not find that murine AZI adequately prevented the AZ1induced degradation of ODC [8]. This may be due to their assay based on 
cotransfections, the effect of which is difficult to control, and thus does not provide ultimate support to their conclusion. Our results quantitatively demonstrate that the binding of ODCp to AZ1 is not only competitive to ODC, but that ODCp also inhibits AZ1 function on ODC degradation.

The interaction with AZ does not induce degradation of AZI [13, 18]. Thus, we wanted to find out whether the binding of ODCp to AZ1 leads to the degradation of ODCp similarly as it does with ODC. On the other hand, if AZ1 has no influence on the degradation of ODCp, the function of ODCp is to act as an AZI. To study this, we used the in vitro degradation assay based on fraction II of reticulocyte lysate (F-II) which is devoid of ubiquitin. Therefore, if any degradation of ODCp was seen in F-II, it is solely induced by the binding of ODCp to AZ1. In the reaction containing ODCp alone or with AZ1, no degradation of ODCp was observed (Figure 3 B). As Bercovich et al. reported earlier [19], in reactions containing ODC and AZ1, ODC was efficiently degraded; providing a positive control for the assay. AZI combined with AZ1 was used as a negative control, and the protein remained undegraded (Figure 3 B). We concluded that the interaction between ODCp and AZ1 does not address ODCp itself to degradation. Instead, the function of ODCp binding to AZ1 is to inhibit the effects of AZ1 on ODC degradation and activity. Consequently, ODCp activates ODC and inhibit its degradation, which emphasizes the role of ODCp as an AZI. The corresponding functional interactions between ODCp and AZ2 or AZ3 remain to be elucidated.

\section{Degradation of ODCp is ubiquitin-dependent}

AZI is degraded via the ubiquitin-proteasome pathway, as are the majority of the cellular proteins [18], while ODC is degraded in an ubiquitin-independent manner mediated by $A Z 1$ [20]. To investigate whether ODCp is degraded by ubiquitination, we conducted an ubiquitin conjugation assay with in vitro translated ${ }^{35} \mathrm{~S}$-labeled proteins. As ubiquitin conjugation plays no part in the turnover of ODC, it was used as a negative control. The use of ODC also monitored the presence of endogenous AZ1 in the lysate. The amounts of both ODCp and AZI diminished as the assay progressed (Figure $4 \mathrm{~A}$ ). As expected, ODC remained undegraded: this also verifies that the reticulocyte lysate itself does not contain enough AZ1 to induce the 
degradation of ODC at selected time scale. It should be noticed that ODC can also be degraded without AZ [21], although this happens at slower rate and thus was not significant in the assay employed. We deduced that ubiquitin conjugation stimulated the degradation of ODCp in reticulocyte lysate. To further confirm this observation, we repeated the ubiquitin conjugation assay in the presence of proteasome inhibitors to see whether high molecular weight ubiquitin-conjugates of ODCp accumulated in the reaction mixture. Ubiquitinated ODCp was clearly observed (Figure 4 B). As expected, AZI was also ubiquitin conjugated, whereas ODC remained unubiquitinated. This ultimately demonstrated that ODCp degradation is ubiquitindependent, as is the degradation of AZI. The finding also demonstrated that ODCp and AZI have similar impacts on the regulation of ODC activity and ODC degradation. This result together with the degradation assays really proves that human ODCp functions as an AZI. We conclude that human ODCp counteracts AZ1 as efficiently as AZI both in vitro and in vivo, and thus represents a novel AZI.

\section{ODCp does not function as a mammalian ADC}

To examine whether ODCp displays L-arginine decarboxylating activity, we used the ADC assay developed by Zhu et al. [4]. As the conditions of the assay were quite extreme $\left(30^{\circ} \mathrm{C}, \mathrm{pH} 8.25\right)$, we repeated the assay also under more physiological conditions $\left(37^{\circ} \mathrm{C}, \mathrm{pH} 7.4\right)$. In neither conditions was ADC activity detected with in vitro translated ODCp or ODC (Figure 5 A). E. coli ADC was used as a positive control, and as shown in Figure $5 \mathrm{~A}$, the assay performed better in $\mathrm{pH} 7.4$ at $37^{\circ} \mathrm{C}$. To investigate the ADC activity of ODCp also in vivo, we transfected COS-7 cells with FLAG tagged ODCp, ODC or empty p3xFLAG-CMV-10 vector. As the ADC activity in rodents was originally measured from mitochondria [22-24], we separated cytosolic and crude mitochondrial fractions from transfectants and measured the ADC activity of them in conditions mentioned above. We also confirmed with anti-FLAG Western blot that the transfectants expressed the fusion proteins (data not shown). No ADC activity was, however, detected in ODCp transfectans in either conditions of the assay (Figure 5 B). As already known, ODC is able to release small quantities of ${ }^{14} \mathrm{CO}_{2}$ from L-arginine converted to ornithine [25], demonstrating that the sensitivity of the assay was sufficient. ADC of E. coli was used as a positive control to show that the assay conditions were permissive for ADC activity. 
To further investigate the L-arginine decarboxylating ability of ODCp, we used liquid chromatography and MALDI-TOF mass spectrometry to quantify agmatine from ODCp transfected COS-7 cells. No generation of agmatine was detected in ODCp expressing COS-7 cells and the sample was negative for agmatine (m/z 131.1291). The limit of detection for agmatine in the spiked sample was $1.5 \mathrm{nmol} / \mathrm{ml}$. The procedure easily measured agmatine from COS-7 cells cultured for $2 \mathrm{~h}$ in the presence of $10 \mathrm{mM}$ agmatine, used as a positive control. The theoretically accurate mass of protonated agmatine is 131.1291 , and the mass accuracy of the sample analyzed was $0.9 \mathrm{mDa}$. The agmatine concentration of the sample was approximately $0.15 \mu \mathrm{mol} / \mathrm{ml}$. For comparison, elevated levels of polyamines were readily detected from ODC transfected COS-7 cells (data not shown).

As the elusive mammalian ADC activity has been suggested to localize in mitochondria [22-24], we also investigated whether expressed FLAG-ODCp travels to the mitochondria. Double immunofluorescent stainings with anti-FLAG and MitoTracker in COS-7 and Paju cells (a human neural crest -derived cell line) transfected with FLAG-ODCp cDNA did not reveal any co-localization (data not shown).

Iyo et al. reported decreased agmatine concentrations in rat neurons transfected with siRNA directed against ODCp [26]. Our present results indicate the binding of ODCp to AZ1-3 which can lead to a drop in the pool of free AZs known to enhance the polyamine uptake [27, 28]. Since agmatine is transported by the same mechanism [29, 30], overexpression of ODCp might lead to an increase in agmatine content. Accordingly, downregulation of ODCp might decrease the content of agmatine. It is thus likely that ODCp influences agmatine concentrations via AZs without any intrinsic ADC activity. The term ADC should now be removed from sequence data banks, since the combined knowledge demonstrates that ODCp has no intrinsic arginine decarboxylating activity. Instead, ODCp is a new member of the AZI family. We agree with López-Contreras et al. that ODCp should be replaced by the term antizyme inhibitor 2 (AZI2) [8]. AZI could thus be renamed as antizyme inhibitor 1 (AZI1). 
ODC and AZI1 are known to be expressed in all types of cells, whereas the expression of ODCp/AZI2 is predominant in brain and testis. The differences in the localization of the two AZI species suggest some functional difference. Assuming that both of these molecules, AZI1 and ODCp/AZI2, are expressed in the same cell, it is intriguing why some cells need such multilevel regulation of ODC activity. It is possible that the function of ODCp/AZI2 is restricted to certain subcellular locations in highly specialized cells in brain and testis.

\section{ACKNOWLEDGMENTS}

We thank Tiiu Arumäe, Anu Harju, and Anna Wilenius for technical assistance. This work was supported by grants from the Academy of Finland, the Finnish Cancer Organisations, the Sigrid Jusélius Foundation, and the Research Foundation of the University of Helsinki.

\section{REFERENCES}

1 Pitkanen, L. T., Heiskala, M. and Andersson, L. C. (2001) Expression of a novel human ornithine decarboxylase-like protein in the central nervous system and testes. Biochem.Biophys.Res.Commun. 287, 1051-1057

2 Heiskala, M., Zhang, J., Hayashi, S., Holtta, E. and Andersson, L. C. (1999) Translocation of ornithine decarboxylase to the surface membrane during cell activation and transformation. EMBO J. 18, 1214-1222

3 Kidron, H., Repo, S., Johnson, M. S. and Salminen, T. A. (2007) Functional classification of amino acid decarboxylases from the alanine racemase structural family by phylogenetic studies. Mol.Biol.Evol. 24, 79-89

4 Zhu, M. Y., Iyo, A., Piletz, J. E. and Regunathan, S. (2004) Expression of human arginine decarboxylase, the biosynthetic enzyme for agmatine.

Biochim.Biophys.Acta. 1670, 156-164

5 Martin-Tanguy, J. (2001) Metabolism and function of polyamines in plants: Recent development (new approaches). Plant Growth Regul. 34, 135-148

6 Nakada, Y. and Itoh, Y. (2003) Identification of the putrescine biosynthetic genes in pseudomonas aeruginosa and characterization of agmatine deiminase and $\mathrm{N}$ carbamoylputrescine amidohydrolase of the arginine decarboxylase pathway.

Microbiology. 149, 707-714 
7 Coleman, C. S., Hu, G. and Pegg, A. E. (2004) Putrescine biosynthesis in mammalian tissues. Biochem.J. 379, 849-855

8 Lopez-Contreras, A. J., Lopez-Garcia, C., Jimenez-Cervantes, C., Cremades, A. and Penafiel, R. (2006) Mouse ornithine decarboxylase-like gene encodes an antizyme inhibitor devoid of ornithine and arginine decarboxylating activity.

J.Biol.Chem. 281, 30896-30906

9 Ivanov, I. P., Gesteland, R. F. and Atkins, J. F. (1998) A second mammalian antizyme: Conservation of programmed ribosomal frameshifting. Genomics. 52, 119129

10 Ivanov, I. P., Rohrwasser, A., Terreros, D. A., Gesteland, R. F. and Atkins, J. F. (2000) Discovery of a spermatogenesis stage-specific ornithine decarboxylase antizyme: Antizyme 3. Proc.Natl.Acad.Sci.U.S.A. 97, 4808-4813

11 Zhu, C., Lang, D. W. and Coffino, P. (1999) Antizyme2 is a negative regulator of ornithine decarboxylase and polyamine transport. J.Biol.Chem. 274, 26425-26430

12 Mangold, U. and Leberer, E. (2005) Regulation of all members of the antizyme family by antizyme inhibitor. Biochem.J. 385, 21-28

13 Murakami, Y., Ichiba, T., Matsufuji, S. and Hayashi, S. (1996) Cloning of antizyme inhibitor, a highly homologous protein to ornithine decarboxylase.

J.Biol.Chem. 271, 3340-3342

14 Nilsson, J., Grahn, B. and Heby, O. (2000) Antizyme inhibitor is rapidly induced in growth-stimulated mouse fibroblasts and releases ornithine decarboxylase from antizyme suppression. Biochem.J. 346 Pt 3, 699-704

15 Lin, Y., Martin, J., Gruendler, C., Farley, J., Meng, X., Li, B. Y., Lechleider, R., Huff, C., Kim, R. H., Grasser, W. A., Paralkar, V. and Wang, T. (2002) A novel link between the proteasome pathway and the signal transduction pathway of the bone morphogenetic proteins (BMPs). BMC Cell Biol. 3, 15

16 Newman, R. M., Mobascher, A., Mangold, U., Koike, C., Diah, S., Schmidt, M., Finley, D. and Zetter, B. R. (2004) Antizyme targets cyclin D1 for degradation. A novel mechanism for cell growth repression. J.Biol.Chem. 279, 41504-41511

17 Li, X. and Coffino, P. (1993) Degradation of ornithine decarboxylase:

Exposure of the C-terminal target by a polyamine-inducible inhibitory protein.

Mol.Cell.Biol. 13, 2377-2383

18 Bercovich, Z. and Kahana, C. (2004) Degradation of antizyme inhibitor, an ornithine decarboxylase homologous protein, is ubiquitin-dependent and is inhibited by antizyme. J.Biol.Chem. 279, 54097-54102

19 Bercovich, Z., Rosenberg-Hasson, Y., Ciechanover, A. and Kahana, C. (1989) Degradation of ornithine decarboxylase in reticulocyte lysate is ATP-dependent but ubiquitin-independent. J.Biol.Chem. 264, 15949-15952 
20 Murakami, Y., Matsufuji, S., Kameji, T., Hayashi, S., Igarashi, K., Tamura, T., Tanaka, K. and Ichihara, A. (1992) Ornithine decarboxylase is degraded by the 26S proteasome without ubiquitination. Nature. 360, 597-599

21 Murakami, Y., Matsufuji, S., Tanaka, K., Ichihara, A. and Hayashi, S. (1993) Involvement of the proteasome and antizyme in ornithine decarboxylase degradation by a reticulocyte lysate. Biochem.J. 295 ( Pt 1), 305-308

22 Li, G., Regunathan, S., Barrow, C. J., Eshraghi, J., Cooper, R. and Reis, D. J. (1994) Agmatine: An endogenous clonidine-displacing substance in the brain. Science. 263, 966-969

23 Lortie, M. J., Novotny, W. F., Peterson, O. W., Vallon, V., Malvey, K., Mendonca, M., Satriano, J., Insel, P., Thomson, S. C. and Blantz, R. C. (1996) Agmatine, a bioactive metabolite of arginine. production, degradation, and functional effects in the kidney of the rat. J.Clin.Invest. 97, 413-420

24 Regunathan, S. and Reis, D. J. (2000) Characterization of arginine decarboxylase in rat brain and liver: Distinction from ornithine decarboxylase. J.Neurochem. 74, 2201-2208

25 Osterman, A. L., Kinch, L. N., Grishin, N. Y. and Phillips, M. A. (1995) Acidic residues important for substrate binding and cofactor reactivity in eukaryotic ornithine decarboxylase identified by alanine scanning mutagenesis. J.Biol.Chem. 270, 11797-11802

26 Iyo, A. H., Zhu, M. Y., Ordway, G. A. and Regunathan, S. (2006) Expression of arginine decarboxylase in brain regions and neuronal cells. J.Neurochem. 96, 10421050

27 Mitchell, J. L., Judd, G. G., Bareyal-Leyser, A. and Ling, S. Y. (1994)

Feedback repression of polyamine transport is mediated by antizyme in mammalian tissue-culture cells. Biochem.J. 299 ( Pt 1), 19-22

28 Sakata, K., Fukuchi-Shimogori, T., Kashiwagi, K. and Igarashi, K. (1997) Identification of regulatory region of antizyme necessary for the negative regulation of polyamine transport. Biochem.Biophys.Res.Commun. 238, 415-419

29 del Valle, A. E., Paz, J. C., Sanchez-Jimenez, F. and Medina, M. A. (2001) Agmatine uptake by cultured hamster kidney cells. Biochem.Biophys.Res.Commun. 280, 307-311

30 Satriano, J., Isome, M., Casero, R. A.,Jr, Thomson, S. C. and Blantz, R. C. (2001) Polyamine transport system mediates agmatine transport in mammalian cells. Am.J.Physiol.Cell.Physiol. 281, C329-34 


\section{FIGURE LEGENDS}

\section{Figure 1. Human ODCp binds to AZ1-3}

A GST pull-down assay to test the binding of ODCp to antizymes. In vitro translated AZ1-3 was pulled down with glutathione sepharose-bound GST or GST-ODCp. The bound material was separated by SDS-PAGE and fluorographed.

Figure 2. Human ODCp acts as an AZI both in vitro and in vivo

(A) The effect of ODCp on the activity of ODC in vitro. ODC, ODCp, AZI and AZ1 were in vitro translated and combined as shown in the figure, after which the ODC activity of the reactions was determined. The experiment was performed in triplicate and the error bars refer to the standard deviation of the results. (B) Influence of ODCp on ODC activity in COS-7 cells. FLAG tagged ODCp, the c-Myc tagged AZI or the empty FLAG-vector were expressed in COS-7 cells. After $24 \mathrm{~h}$, the ODC activity of cell lysates was determined. The bars represent mean values of two simultaneous transfections. The measurements were performed as triplicate and the error bars refer to the standard deviation of the mean.

\section{Figure 3. Human ODCp inhibits AZ1-induced degradation of ODC}

(A) In vitro degradation assay to study the effect of ODCp on the AZ1-induced degradation of ODC. In vitro translated proteins were combined as indicated and incubated in ATP-regenerating buffer as described in "Experimental". The reactions were resolved by SDS-PAGE and fluorographed. Because the molecular masses of ODC, AZI and ODCp are nearly equal, AZI and ODCp were produced as unlabeled to avoid mixing them up with ODC. The amount of labeled AZ1 (not shown) was used to control the amount of protein loaded on the gel in the parallel samples. (B) In vitro degradation assay of ODCp in F-II which lacks ubiquitin. In vitro translated proteins were combined as shown in the illustration, incubated in buffer with F-II and resolved by SDS-PAGE and fluorography.

\section{Figure 4. Degradation of ODCp is ubiquitin-dependent}

(A) Ubiquitin conjugation assay of ODCp used to test the degradation of ODCp. In vitro translated AZI, ODCp and ODC were incubated in a buffer containing the 
complete ubiquitin conjugating system. The samples were fractionated by SDS-PAGE and fluorographed. (B) Ubiquitin conjugation assay with proteasome inhibitors. ${ }^{35} \mathrm{~S}$ labeled AZI, ODCp and ODC were incubated in an ubiquitin conjugating buffer with MG132 and clasto-Lactacystin $\beta$-lactone. Ubiquitination of equal-sized aliquots was determined by SDS-PAGE and fluorography.

\section{Figure 5. Human ODCp has no intrinsic ADC activity}

(A) The ADC activity measurement of in vitro translation products. ODC, ODCp, and empty pCDNA3.1 vector were in vitro translated and the ADC activity of the samples was determined in $\mathrm{pH} 7.4$ at $37{ }^{\circ} \mathrm{C}$ or in $\mathrm{pH} 8.25$ at $30{ }^{\circ} \mathrm{C}$. E. coli ADC was used as positive control. In both conditions the experiment was performed in triplicate and the error bars refer to the standard deviation of the mean. (B) Activity of ADC in COS-7 cells expressing FLAG-ODC, FLAG-ODCp or the empty p3xFLAG-CMV-10 vector. Cytosolic and crude mitochondrial fractions of the transfectants were obtained as described in "Experimental" and the ADC activity was measured in $\mathrm{pH} 7.4$ at $37^{\circ} \mathrm{C}$ or in $\mathrm{pH} 8.25$ at $30{ }^{\circ} \mathrm{C}$. The measurement was performed in triplicate and the error bars refer to the standard deviation of the mean. Note the logarithmic scale on the vertical axis. *The activity of E. coli ADC, serving as positive control, is indicated in $\mathrm{C}^{14} \mathrm{cpm} / \mathrm{U} / \mathrm{h}$. 
Figure 1. 


\section{Figure 2.}

A

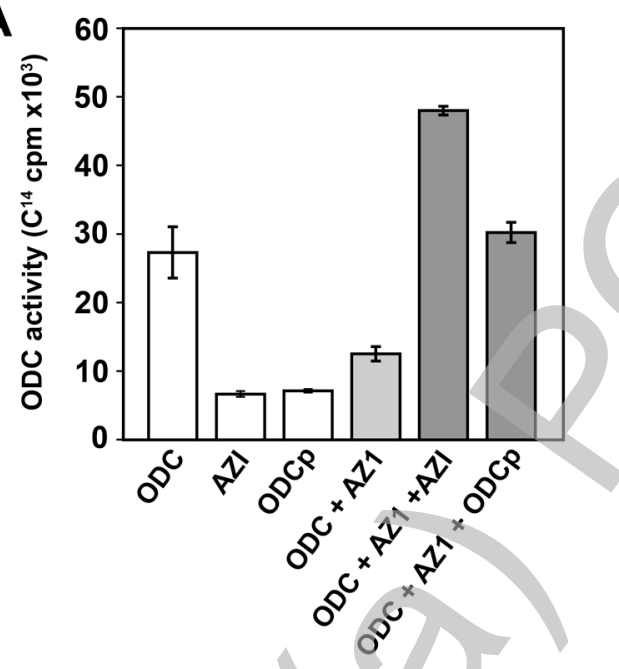

B

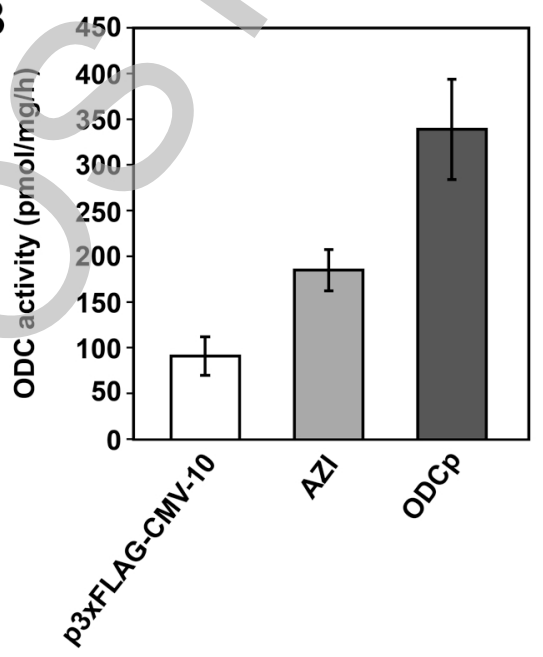


Figure 3.

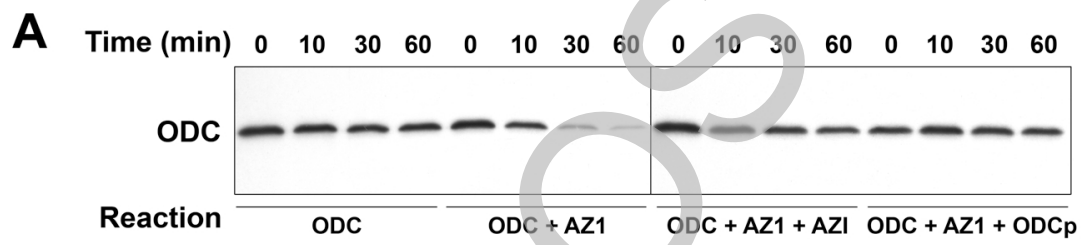

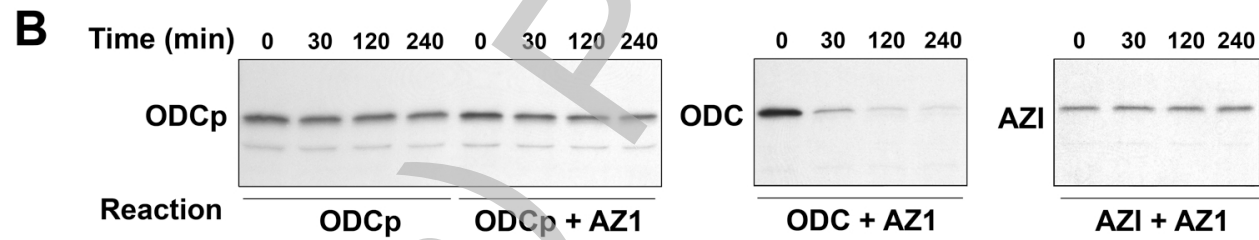


Figure 4.
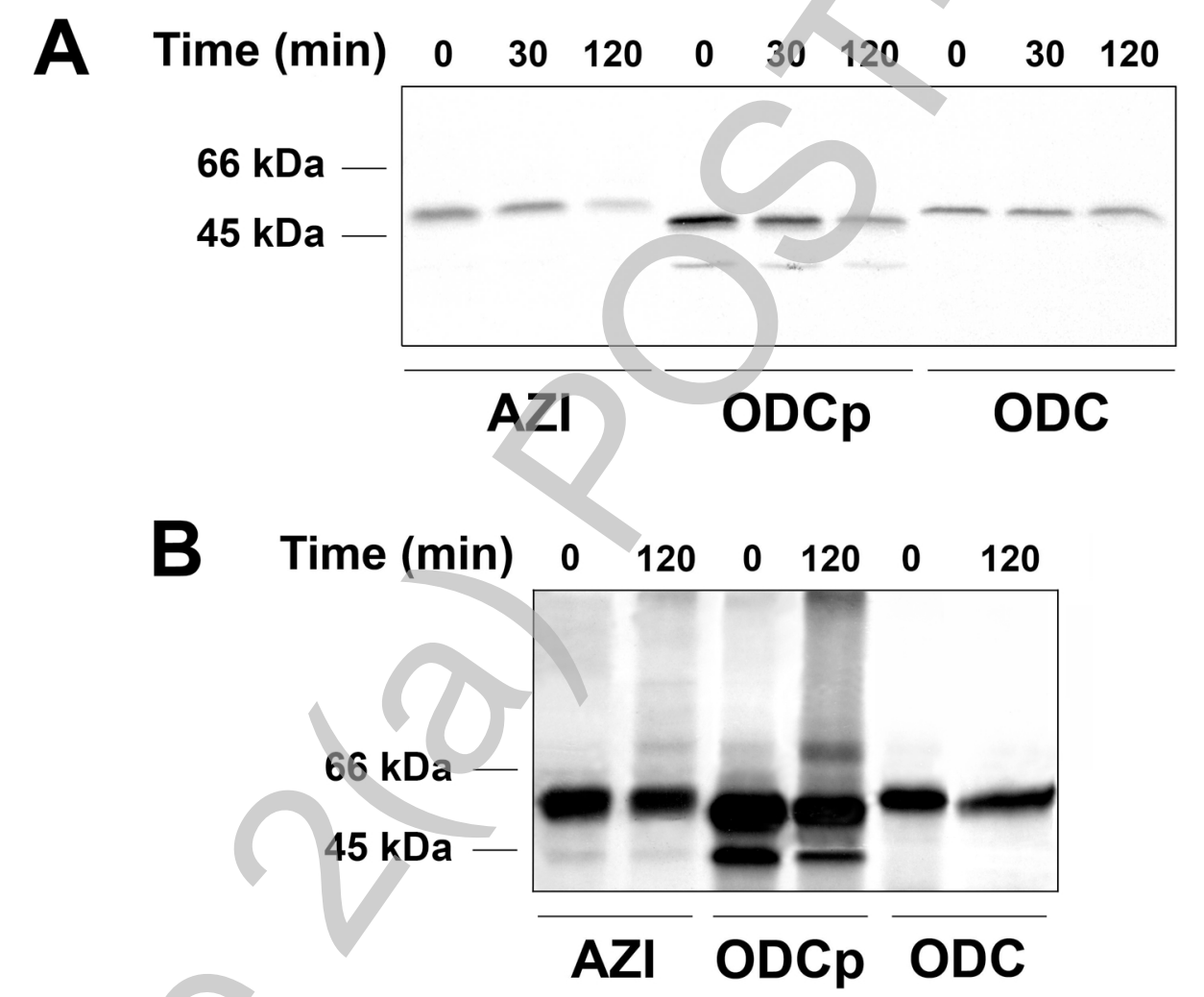
Figure 5.

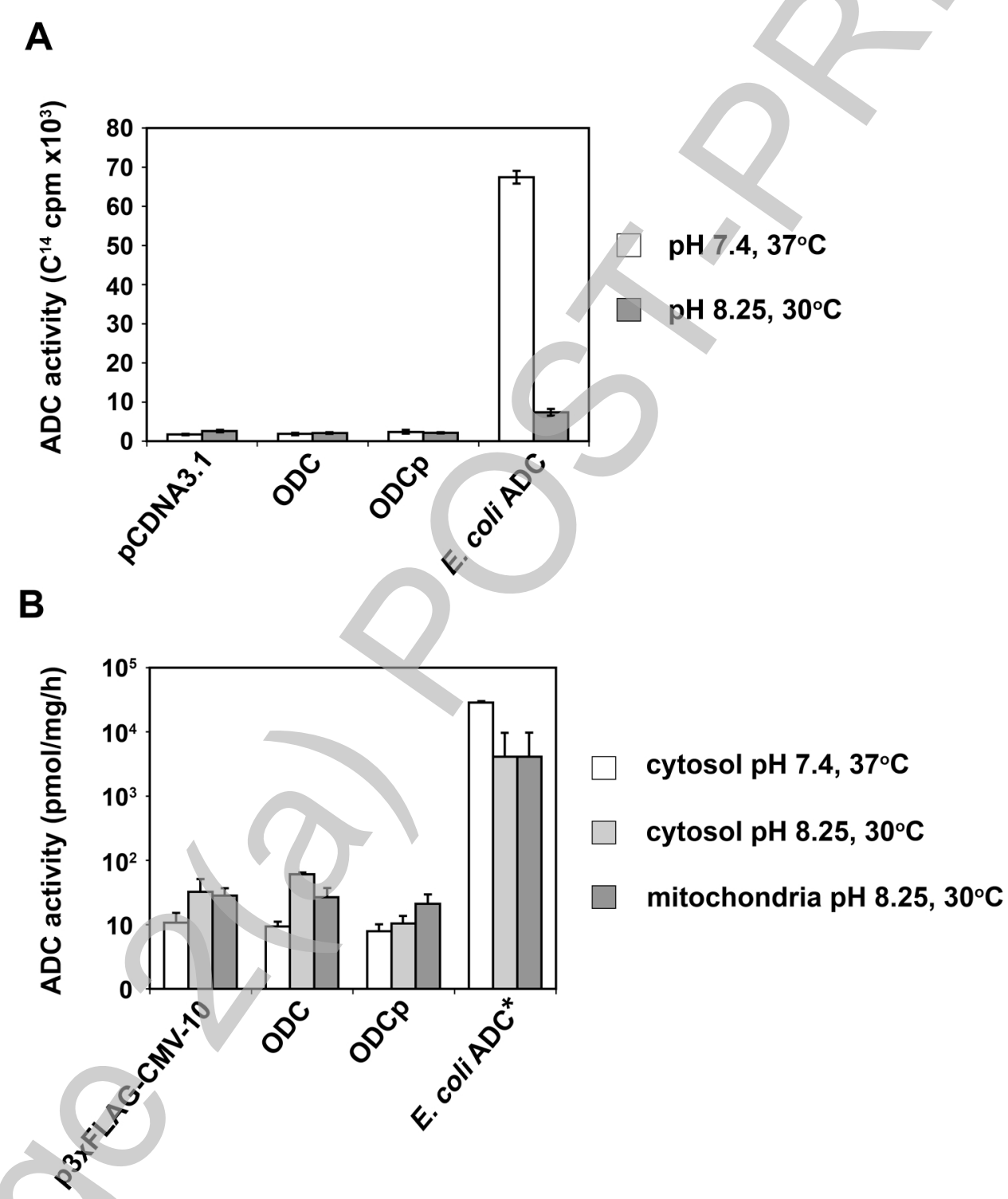

